\title{
ESTIMATION OF THE ATTITUDE PERTURBANCE USING PARALLAX IMAGERY - APPLICATION TO ZY-3 SATELLITE
}

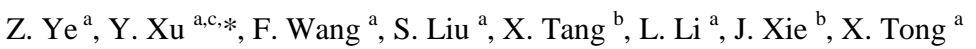 \\ ${ }^{\text {a }}$ College of Surveying and Geo-Informatics, Tongji University, 200092, Shanghai, P. R. China - (89_yezhen, liusjtj, \\ xhtong)@tongji.edu.cn, wangfx1228@sina.com, li_lingyun@yeah.net \\ ${ }^{\mathrm{b}}$ Satellite Surveying and Mapping Application Center, National Administration of Surveying, Mapping and Geoinformation, \\ 101300, Beijing, P. R.China - txm@sasmac.cn, junfeng_xie@163.com \\ ${ }^{c}$ Photogrammetry and Remote Sensing, Technische Universität München, 80333, München, Germany - yusheng.xu@tum.de
}

KEY WORDS: Attitude Perturbance, ZY-3 Satellite, Parallax Observation, Multispectral Images, Image Matching

\begin{abstract}
:
The inflight stability of the earth observation satellite is a significant factor having an influence on the geometric performance of its imagery. The estimation of the attitude perturbance is therefore indispensable to spaceborne photogrammetry application. In this paper, an experiment using a short strip of multispectral images obtained from ZY-3 satellite is conducted. The detection method for attitude perturbance based on parallax observation is adopted, while a novel approach of estimating the attitude perturbance angles from the perturbance caused image displacements is also proposed and demonstrated. The estimated results indicate that the ZY-3 satellite was affected by considerable perturbances with distinctive frequencies during the flight of the investigated images, and attitude perturbances hava also undermined the geometric performance of the acquired imagery.
\end{abstract}

\section{INTRODUCTION}

The attitude perturbance, which is commonly induced by the dynamic structure on board, the changes in the body temperature, the operation of the orbital control, and so on, is a universal problem occurring in the flight of earth observation satellites (Amberg et al., 2013; Johnston and Thornton, 2000; Takaku and Tadono, 2010; Iwasaki, 2011). With the increase of spatial resolution and the enhancement of maneuverability, the attitude perturbance of the satellite platform has made a crucial impact upon geometric performance of high resolution satellite images (HRSI) (Liu et al., 2011; Mattson et al., 2009; Poli et al., 2014). With the help of the commonly used high resolution CCD linear array sensors, the attitude perturbance can be effectively extracted from the HRSI obtained by an imaging system with parallax observation (Amberg et al., 2013; Iwasaki, 2011). Some successful application has already been conducted on typical observation satellites such as High Resolution Imaging Science Experiment (HiRISE) (Mattson et al., 2009), Lunar Reconnaissance Orbiter (LRO) (Mattson et al., 2010; Mattson et al., 2011), Terra (Iwasaki, 2011), SPOT 5 (Latry and Delvit, 2009), Pleiades-HR (Amberg et al., 2013). Compared with the typical approaches, the methods of parallax observation seem to be more economically and technically feasible for many on-orbit satellites.

Launched on January 9, 2012, ZY-3 is China's first civilian stereo surveying and mapping satellite (Jiang et al., 2014). It is equipped with one three-line-array panchromatic stereo camera and one multispectral camera, with their ground resolution reaching $2.1 \mathrm{~m}$ (nadir), $3.5 \mathrm{~m}$ (forward and backward) and $5.8 \mathrm{~m}$, respectively (Pan et al., 2013), but the sampling rate of the attitude sensors onboard ZY-3 is only $4 \mathrm{~Hz}$.

To optimize the performance of attitude sensors and minimize attitude perturbances, in this paper, a precise estimation of the attitude perturbance of ZY-3 satellite is carried out. The strategy includes three steps depicted in Figure 1. Firstly, the disparity map is computed by dense matching using phase correlation algorithm. Secondly, the parallax disparities are obtained from the refined disparity maps and then the image displacements caused by the perturbance are calculated from parallax disparity. Finally, the attitude perturbance angle is extracted from the image displacements and estimated with the auxiliary file and coordinate transformation.

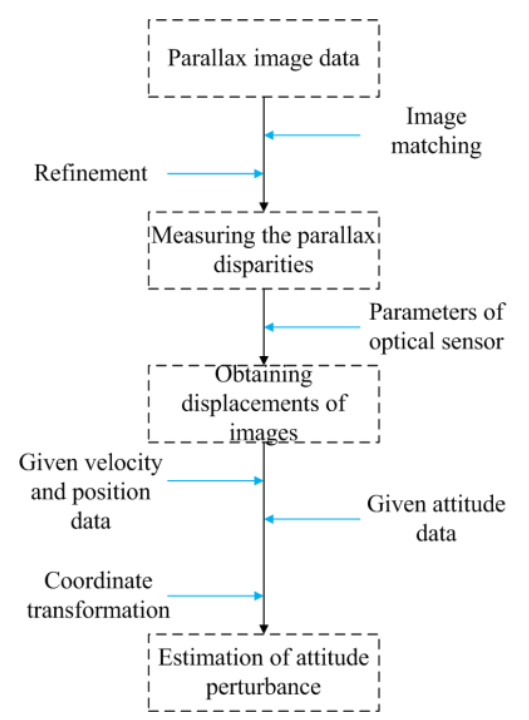

Figure 1. Schematic view of estimating the attitude perturbance in this study

\footnotetext{
* Corresponding author.
} 


\section{PARALLAX DETERMINATION SYSTEM OF THE ZY-3 SATELLITE}

The parallax determination system of the ZY-3 satellite is quite similar to that of the ASTER sensors (Iwasaki, 2011), which derives from its focal plane geometry of multispectral sensors as presented in (Jiang et al., 2014). With regard to ZY-3, the distance from Band1(blue) to Band2(green) is 152 lines, while the distances from Band2(green) to Band3(red) and from Band3(red) to Band4(NIR) are both 128 lines (Pan et al., 2013), with their parallax intervals reaching $121.6 \mathrm{~ms}$ and $102.4 \mathrm{~ms}$, respectively. Since the attitude perturbance may give rise to the motion of sensors, there will be the tiny differences in the geometric location of the identical features on parallax images. By measuring these differences, namely the parallax disparity between two parallax images of adjacent bands, the attitude perturbance can be deduced and estimated.

\section{IMAGE CORRELATION FOR THE PARALLAX DISPARITY}

To measure the parallax disparity accurately, a phase correlation method on the basis of Peak Evaluation Formula (PEF) (Nagashima et al., 2006) and a dense matching strategy (Morgan et al., 2010) are employed, with the matching accuracy reaching the sub-pixel level. As the parallax disparities achieved by the dense matching is merely a relative estimate of image displacement resulted from attitude perturbance, an approach utilizing the translation properties of the Fourier transform is adopted (Iwasaki, 2011), in order to solve the deconvolution problem of (1).

$$
\varphi(t)=s(t)-s(t-\delta)
$$

where

$$
\begin{gathered}
\varphi(t)=\text { the relative parallax disparity } \\
s(t)=\text { the absolute image displacement } \\
\delta=\text { the time lag between different bands }
\end{gathered}
$$

Figure 2 illustrates the flowchart of measuring the parallax disparities by the use of image correlation approaches and the calculation of the image displacements.

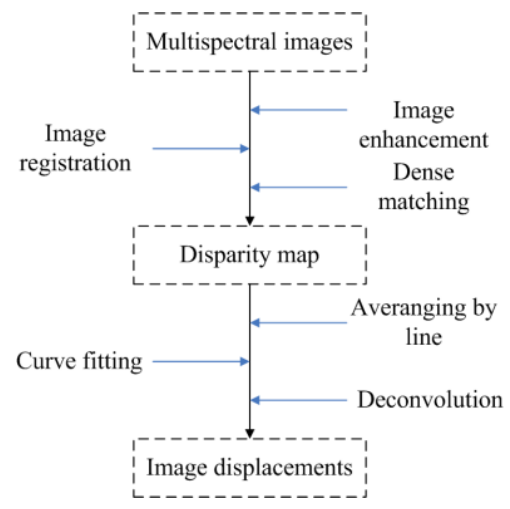

Figure 2. The flowchart of obtaining the parallax disparities and image displacements

\section{ESTIMATION OF THE ATTITUDE PERTURBANCE}

The image displacement deduced from the parallax disparity is only the quantitive impact of the attitude perturbance on the imagery. Therefore, the displacement of images should be converted to the perturbance angles of the platform. The given attitude data of the ZY-3 satellite in the auxiliary file, which has been preprocessed (Zhang et al. 2014) and given in the form of three Eula angles, are the rotation angles from the body coordinate frame to the orbit coordinate frame (Jiang et al., 2014; Pan et al., 2013; Zhang et al. 2014). Nevertheless, the estimated perturbance angles of the platform is projected from the body coordinate frame to the orbit coordinate frame, which depicts the tiny rotation angles between the body coordinate frame and the orbit coordinate frame. Therefore, a coordinate transformation is required to obtain the attitude perturbance in the orbit coordinate frame system, which can directly reflect the influence of the attitude perturbance on the flight.

With interior and exterior orientation elements, the typical collinearity equation of the imaging model of ZY-3 satellite can be represented as (2) (Jiang et al., 2014; Pan et al., 2013):

$$
\left[\begin{array}{l}
X \\
Y \\
Z
\end{array}\right]=\left[\begin{array}{c}
X_{0} \\
Y_{0} \\
Z_{0}
\end{array}\right]+\lambda \cdot R_{\text {sensor }}^{E C F} \cdot\left[\begin{array}{c}
\tan \left(\psi_{y}\right) \\
-\tan \left(\psi_{x}\right) \\
1
\end{array}\right]
$$

where

$$
\begin{aligned}
& \lambda=\text { the scale factor } \\
& \psi_{x}, \psi_{y}=\text { the interior orientation elements } \\
& X_{0}, Y_{0}, Z_{0}=\text { the satellite position } \\
& X, Y, Z=\text { the ground position of the object } \\
& R_{\text {sensor }}^{E C F}=\text { the rotation matrix }
\end{aligned}
$$

$R_{\text {sensor }}^{E C F}$ is the rotation matrix between the sensor coordinate frame and ECF coordinate frame, which can be decomposed into three steps

$$
\begin{gathered}
R_{\text {sensor }}^{E C F}=R_{\text {orbit }}^{E C F} \cdot R_{\text {body }}^{\text {orbit }} \cdot R_{\text {sensor }}^{\text {body }} \\
R_{\text {body }}^{\text {orbit }}=R(\omega) \cdot R(\varphi) \cdot R(\kappa)
\end{gathered}
$$

where $\quad R_{\text {orbit }}^{E C F}=$ the transformation from the orbit coordinate frame to the ECF coordinate frame

$$
R_{\text {sensor }}^{\text {body }}=\text { the rotation matrix from the sensor }
$$

coordinate frame to the body coordinate frame $R_{b o d y}^{\text {orbit }}=$ the rotation matrix from the body coordinate

frame to the orbit coordinate frame $\omega, \varphi, \kappa=$ the rotation angles from the body coordinate frame to the orbit coordinate frame

The $R_{\text {sensor }}^{\text {body }}$ is generally achieved from on-orbit calibrated parameters from the optical sensor to the spacecrafts, while $R_{\text {body }}^{\text {orbit }}$ is the rotation matrix through the given attitude data in the roll, pitch and yaw direction, which converts the body coordinate frame to the orbit coordinate frame. The attitude perturbance can be regarded as unexpected errors of these given attitude angles, in the form of $(\Delta \omega, \Delta \varphi, \Delta \kappa)$.

Considering that the perturbances are commonly quite small, the displacements of images can be counted as perturbance angles of the line of sight vector (LOS) in the sensor coordinate 
frame, $\Delta \overline{\psi_{x}}$ and $\Delta \overline{\psi_{y}}$, which are calculated by the following equations.

$$
\begin{aligned}
\Delta \overline{\psi_{x}} & =\frac{\overline{S_{x}} \cdot \sigma}{f_{c}} \\
\Delta \overline{\psi_{y}} & =\frac{\overline{S_{y}} \cdot \sigma}{f_{c}}
\end{aligned}
$$

where $\overline{S_{x}}, \overline{S_{y}}=$ image displacements averaged by line in the $\mathrm{x}$ - and $\mathrm{y}$ - directions

$$
\begin{aligned}
& \sigma=\text { the size of the CCD elements } \\
& f_{c}=\text { the focal length }
\end{aligned}
$$

Thus, the relationship between the perturbance angle of the LOS and the attitude perturbance angle can be expressed as follows:

$$
\begin{aligned}
& R(\omega+\Delta \omega) \cdot R(\varphi+\Delta \varphi) \cdot R(\kappa+\Delta \kappa) \cdot R_{\text {sensor }}^{\text {body }} \\
& =R(\omega) \cdot R(\varphi) \cdot R(\kappa) \cdot R_{\text {sensor }}^{\text {body }} \cdot R\left(\Delta \overline{\psi_{x}}\right) \cdot R\left(\Delta \overline{\psi_{y}}\right)
\end{aligned}
$$

With the help of (6), the values of the attitude perturbance can be estimated from the image displacements.

\section{INVESTIGATION RESULT}

In this paper, a short strip of 12 scenes of multispectral images was used to analyse and estimate the attitude perturbance along a flight orbit. The multispectral image data used in the experiment was a Level 0 dataset, which were merely processed with radio corrections. Figure 3 shows the distribution of the investigated image data. The image data of orbit 5619 is acquired on Jan. 12, 2013, and the scenes used are from 7-124 to $7-135$. The study area covered by the image data is located in the central area of China, ranging from the southern Inner Mongolia Province to the northern Henan Province. The image bands used are the Band 2 and the Band 3.

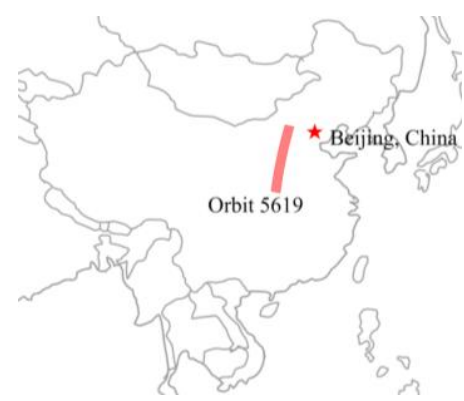

Figure 3. The distribution of the experimental images

Figure 4 shows an illustration of the disparity maps of 3 scenes in the short strip images of orbit 5619. In the disparity maps, the light stripes appearing in the CCD array direction depict the changes of parallax disparities caused by the unexpected motions of the sensor arrays, which is corresponding to the attitude perturbance. From the intervals between these periodical stripes, we can extract the frequency of the perturbance, and by measuring the degree of gray level in the disparity map, we can also estimate the amplitude of the parallax disparities. It is noticeable that there exist clear topographic patterns in the disparity maps in the along-track direction inducing by the tiny stereo vision of the adjacent multispectral bands. They reflect the changes in elevation due to the mountainous areas in the image scene (Arai and Iwasaki, 2012).

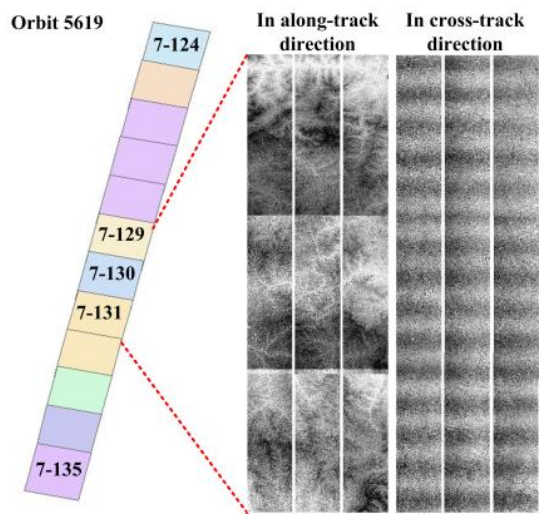

Figure 4 . The disparity maps of the experimental images

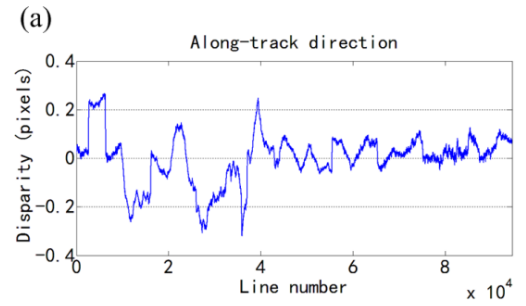

(b)

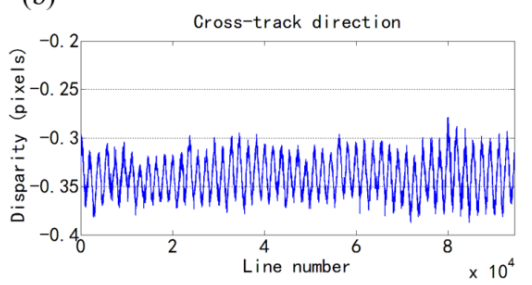

Figure 5. The mean parallax disparities of the 12 experimental images

(a)

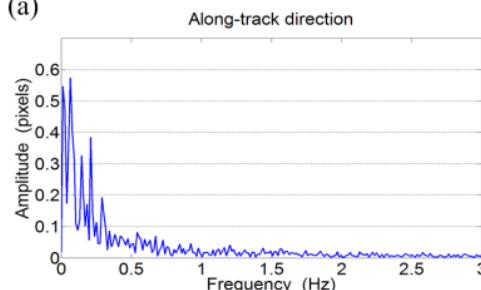

(b)

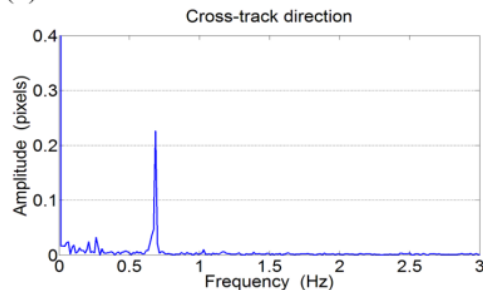

Figure 6. The spectrograms of the mean parallax disparities

The plots in Figures 5 (a) and (b) depict the averaged parallax disparities at each line of the whole 12 scenes in the short strip, while the spectrograms of these parallax disparities are given in 
Figs. 6 (a) and (b). It can be seen from the figures that the parallax disparities in the cross-track direction reveal an obvious periodical trend, but they are affected by topographic patterns and show no significant tendency in the along-track direction. Considering the ground resolution of the multispectral bands is approximately $5.8 \mathrm{~m}$, the perturbance angles of LOS can be estimated from image displacements by the use of Eq. (5). The corresponding attitude perturbance values are also calculated by the use of Eq. (6). The given attitude data utilized in the Eq. (6) was smoothed to eliminate the high-frequency component and the drift angle in the yaw angle was also separated. In Figures 7 and 8, an instance of a single scene in the whole strip illustrating parallax disparities, image displacements and the estimated attitude perturbance values was given. Apparently, there are two possible components of different frequency exhibiting by the image displacements caused by the attitude perturbances, with their frequencies reaching approximately $0.256 \mathrm{~Hz}$ and $0.694 \mathrm{~Hz}$, respectively.
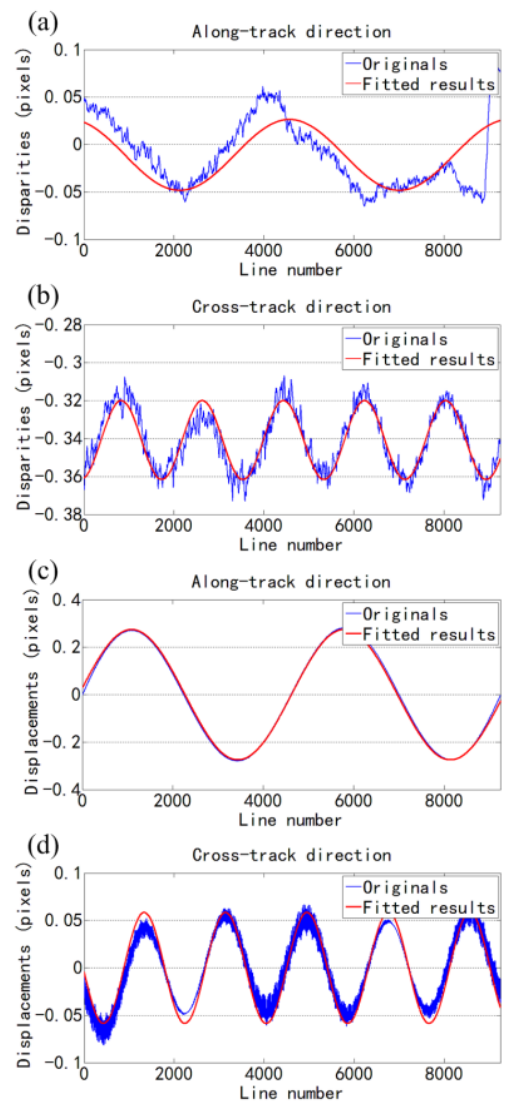

Figure 7. An example of scene Orbit 5619-7-130 illustrates the case of the estimated results. The mean parallax disparity obtained with Band 2 and Band 3 in (a) the along-track directionand (b) the cross-track direction. The image displacement caused by attitude perturbance in (c) the alongtrack directionand (d) the cross-track direction

As expected, the Figure 8 exhibits the periodical attitude perturbances with two distinctive frequencies in the roll, pitch and yaw directions in the orbit coordinate frame. Theoretically, these attitude perturbances, which can be as large as approximately 0.8 second of arc, are adverse to the accurate geometric mapping and surveying. It is of great value to find ways to eliminate its negative influences.
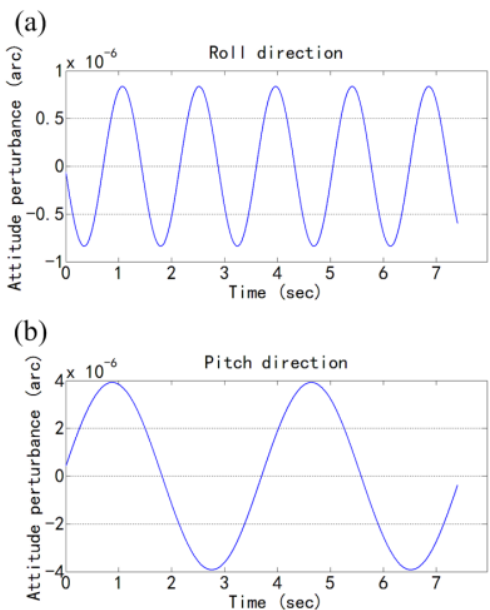

(c)

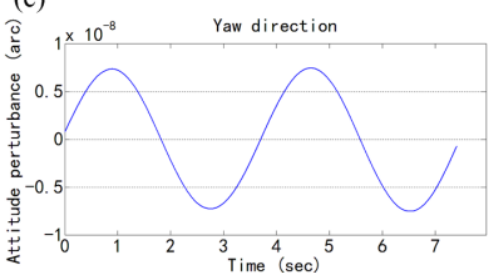

Figure 8. An instance of the scene Orbit 5619-7-130 showing the estimated attitude perturbance angle

\section{CONCLUSION}

The estimation of the attitude perturbance of the earth observation satellite is of great value to improve the performance of high-resolution satellite imagery. In the case of ZY-3 satellite, the effective estimation approach for the attitude perturbance based on parallax observation was adopted to characterize the attitude perturbance information. With the use of a short strip of 12 scenes of multispectral images, it is demonstrated that there exist attitude perturbances consisting of two components with different frequency and amplitude, which have a considerable impact on the attitude stability in all three directions. However, the specific cause of the attitude perturbance has not yet been determined and need to be further analysed. Moreover, the compensation of the influence of the perturbance as well as its detailed characteristics require further investigations in the future as well.

\section{REFERENCES}

Amberg, V., Dechoz, C., Bernard, L., Greslou, D., De Lussy, F. and Lebegue, L., 2013. In-flight attitude perturbances estimation: application to PLEIADES-HR satellites. In: Proc. SPIE 8866, Earth Observing Systems XVIII, California, USA, Sept. 2013, pp. 121-129.

Arai, T. and Iwasaki, A., 2012. Fine image matching for narrow baseline stereovision. In: Proc. of 2012 IEEE Int. Geoscience and Remote Sensing Symp., pp. 2336-2339.

Iwasaki, A., 2011. Detection and estimation of satellite attitude jitter using remote sensing imagery. In: Hall, J. (Ed.), Advances in Spacecraft Technologies, InTech, pp. 257-272. 
Jiang, Y., Zhang, G., Tang, X., Li, D., Huang, W. and Pan, H., 2014. Geometric Calibration and Accuracy Assessment of ZiYuan-3 Multispectral Images. IEEE Trans. Geosci. Remote Sens., 52, (7), pp. 4161-4172.

Johnston, J. D. and Thornton, E. A., 2000. Thermally induced dynamics of satellite solar panels. J. Spacecraft. Rockets., 37(5), pp. 604-613.

Liu, S., Fraser, C. S., Zhang, C., Ravanbakhsh, M. and Tong, X., 2011. Georeferencing performance of THEOS satellite imagery. Photogramm. Rec., 26(134), pp. 250-262.

Latry, C. and Delvit, J.-M., 2009. Staggered arrays for high resolution earth observing systems. In: Proc. SPIE, Earth Observing Systems XIV, San Diego, CA, USA, pp. 1-12.

Mattson, S., Bartels, A., Boyd, A., Calhoun, P., Hsu, O., McEwen, A., Robinson, M., Siskind, J. and Tran, T., 2011. Continuing analysis of spacecraft jitter in LROC-NAC. In: Proc. of Lunar and Planetary Science Conference XLI. The Woodlands, TX, USA, pp. 1871.

Mattson, S., Boyd, A., Kirk, R. L., Cook, D. A. and HowingtonKraus, E., 2009. HiJACK: Correcting spacecraft jitter in HiRISE images of Mars. In: Proc. Eur. Planet. Sci. Conf. Potsdam, Germany, 2009.

Mattson, S., Robinson, M., McEwen, A., Bartels, A., BowmanCisneros, E., Li, R., Lawver, J., Tran, T., Paris, K. and the LROC Team, 2010. Early assessment of spacecraft jitter in LROC-NAC. In: Proc. of Lunar and Planetary Science Conference XXXXI.

Morgan, G. L. K., Liu, J. G. and Yan, H., 2010. Precise subpixel disparity measurement from very narrow baseline stereo. IEEE Trans. Geosci. Remote Sens., 48( 9), pp. 34243433.

Nagashima, S., Aoki, T., Higuchi, T. and Kobayashi, K., 2006. A subpixel image matching technique using phase-only correlation. In: IEEE Int. Symp. Intelligent Signal Processing and Communications Systems, pp. 701-704.

Pan, H., Zhang, G., Tang, X., Li, D., Zhu, X., Zhou, P. and Jiang, Y., 2013. Basic products of the ZiYuan-3 satellite and accuracy evaluation, Photogramm. Eng. Remote Sens., 79(12), pp. 1131-1145.

Poli, D., Remondino, F., Angiuli, E. and Agugiaro, G., 2014. Radiometric and geometric evaluation of GeoEye-1, WorldView-2 and Pléiades-1A stereo images for 3D information extraction. ISPRS J. Photogramm. Remote Sens..

Takaku, J. and Tadono, T., 2010. High resolution DSM generation from ALOS PRISM-processing status and influence of attitude fluctuation. In: Proc. of 2010 IEEE Int. Geoscience and Remote Sensing Symp., pp. 4228-4231.

Takaku, J. and Tadono, T., 2011. RPC generations on ALOS PRISM and AVNIR-2. In: Proc. of 2011 IEEE Int. Geoscience and Remote Sensing Symp., pp. 539-542.

Zhang, Y., Zheng, M., Xiong, J., Lu, Y. and Xiong, X., 2014. On-orbit geometric calibration of ZY-3 three-line array imagery with multistrip data sets. IEEE Trans. Geosci. Remote Sens., 52(1), pp. 224-234. 